Article

\title{
Human Papillomavirus E6/E7-Specific siRNA Potentiates the Effect of Radiotherapy for Cervical Cancer in Vitro and in Vivo
}

\author{
Hun Soon Jung ${ }^{1,2, \dagger}$, Nirmal Rajasekaran ${ }^{1, \dagger}$, Sang Yong Song ${ }^{3}$, Young Deug Kim ${ }^{2}$, \\ Sungyoul Hong ${ }^{1}$, Hyuck Jae Choi ${ }^{4}$, Young Seok Kim ${ }^{5}$, Jong-Sun Choi ${ }^{6}$, Yoon-La Choi ${ }^{3}$ \\ and Young Kee Shin 1,6,7,*
}

1 Research Institute of Pharmaceutical Science, Department of Pharmacy, College of Pharmacy, Seoul National University, Seoul 151-742, Korea; E-Mails: hunsoonjung@abionbio.com (H.S.J.); nirmalpharma@gmail.com (N.R.); sungyoul@snu.ac.kr (S.H.)

2 ABION Inc. R\&D Center, 9th Floor, HanWha Biz Metro Bldg, 242 Digital-ro, Guro-gu, Seoul 152-733, Korea; E-Mail: todnos@abionbio.com

3 Department of Pathology, Samsung Medical Center, Sungkyunkwan University School of Medicine, Seoul 135-710, Korea; E-Mails: yodasong@hanmail.net (S.Y.S.); yla.choi@samsung.com (Y.-L.C.)

4 Department of Radiology, Kangwon National University Hospital, Kangwon-do 200-722, Korea; E-Mail: choihjmd@gmail.com

5 Department of Radiation Oncology, Asan Medical Center, College of Medicine, University of Ulsan, Seoul 138-736, Korea; E-Mail: ysk@amc.seoul.kr

6 The Center for Anti-cancer CDx, N-Bio, Seoul National University, Seoul 151-742, Korea; E-Mail: jscmd@naver.com

7 Tumor Microenvironment Global Core Research Center, Seoul National University, Seoul 151-742, Korea

$\uparrow$ These authors contributed equally to this work.

* Author to whom correspondence should be addressed; E-Mail: ykeeshin@snu.ac.kr; Tel.: +82-2-880-9126; Fax: +82-2-872-1795.

Academic Editor: Michael R. Ladomery

Received: 10 March 2015 / Accepted: 12 May 2015 / Published: 29 May 2015

\begin{abstract}
The functional inactivation of TP53 and Rb tumor suppressor proteins by the HPV-derived E6 and E7 oncoproteins is likely an important step in cervical carcinogenesis. We have previously shown siRNA technology to selectively silence both E6/E7 oncogenes and demonstrated that the synthetic siRNAs could specifically block its
\end{abstract}


expression in HPV-positive cervical cancer cells. Herein, we investigated the potentiality of E6/E7 siRNA candidates as radiosensitizers of radiotherapy for the human cervical carcinomas. HeLa and SiHa cells were transfected with HPV E6/E7 siRNA; the combined cytotoxic effect of E6/E7 siRNA and radiation was assessed by using the cell viability assay, flow cytometric analysis and the senescence-associated $\beta$-galactosidase (SA- $\beta$-Gal) assay. In addition, we also investigated the effect of combined therapy with irradiation and E6/E7 siRNA intravenous injection in an in vivo xenograft model. Combination therapy with siRNA and irradiation efficiently retarded tumor growth in established tumors of human cervical cancer cell xenografted mice. In addition, the chemically-modified HPV16 and $18 \mathrm{E} 6 / \mathrm{E} 7$ pooled siRNA in combination with irradiation strongly inhibited the growth of cervical cancer cells. Our results indicated that simultaneous inhibition of HPV E6/E7 oncogene expression with radiotherapy can promote potent antitumor activity and radiosensitizing activity in human cervical carcinomas.

Keywords: E6; E7; siRNA; Concurrent Chemoradiation therapy (CCRT); radiotherapy; radiosensitizer; cervical cancer

\section{Introduction}

Cervical cancer is the second most common cancer in women worldwide. Infection of the cervix with high-risk human papillomavirus (HPV), such as types 16 or 18, is a major cause of cervical cancer. The E5, E6 and E7 oncoproteins encoded by HPV play a critical role in cervical carcinoma [1-3]. Although most HPV-associated cervical carcinomas, unlike many other cancers, carry the wild-type TP53 gene, the levels of TP53 protein in these carcinomas remain remarkably low, because the protein is constantly targeted for degradation by the E6 viral protein [4,5]. In addition, the E7 binds to the retinoblastoma $(\mathrm{RB})$ family of tumor suppressor proteins and disrupts $\mathrm{RB} / \mathrm{E} 2 \mathrm{~F}$ complexes, thereby driving cell division [6]. The functional inactivation of TP53 and RB tumor suppressor proteins by the HPV-derived E6 and E7 oncoproteins is likely an important step in cervical carcinogenesis. Thus, the E6 and E7 proteins may be suitable targets for treating cervical cancer. The HPV16 E5 is a hydrophobic protein observed in the endoplasmic reticulum, Golgi apparatus and nuclear membrane of infected cells. The E5 oncoprotein displays transforming activity and is believed to enhance the oncogenic effect of E6 and E7. However, its mechanistic role is not clear during cervical carcinogenesis [7,8].

Recently, RNA interference (RNAi) has been developed as a novel therapeutic strategy and is currently in early stage clinical trials [9]. Many investigators have developed RNAi targeting E6 or E6/E7, which promote the accumulation of TP53 and/or hypo-phosphorylated RB (pRB). This eventually leads to the induction of apoptosis and/or senescence in cervical carcinoma cells [10-16]. Previously, we reported that small interfering RNA (siRNA) targeting E6/E7 in combination with cisplatin (cis-diamminedichloroplatinum II; CDDP) exerts a synergistic effect through the restoration of TP53 and RB/E2F; moreover, it has a more potent therapeutic effect for cervical cancer than either of the agents alone have [17]. Thus far, we have validated the E6/E7 siRNA as a potential 
chemosensitizer for use in cervical cancer. Recent studies have shown that chemotherapy effectively enhances radiosensitivity in cervical cancer, and CDDP enhances the radiosensitivity of HPV16-positive SiHa cells [18]. Published reports have shown that concurrent chemoradiation therapy (CCRT) restored the function of TP53 at a molecular level. Arsenic trioxide (As2O3) [19] and ubenimex, a aminopeptidase $\mathrm{N}(\mathrm{APN}) / \mathrm{CD} 13$ inhibitor [20] are also known to enhance the radiosensitivity of human cervical carcinoma cells in vitro and in vivo. Randomized clinical trials for cervical cancer have shown that concomitant treatment with CDDP and radiotherapy (RT) is better than RT alone at improving prognosis [21-23]. Therefore, CCRT is recommended as the first-line therapy for patients with stage II B or a higher stage disease, and it is also recommended as an adjuvant therapy following radical hysterectomy for patients with locally-advanced cervical cancer [24,25]. This practice guideline by CCRT has improved survival rates in patients with cervical cancer. However, whether synergistic therapeutic effects can be achieved by combination therapy with E6/E7 silencing and RT remains to be determined. In the present study, we assessed the synergistic therapeutic effects of combination therapy with E6/E7 silencing and RT in HPV-positive cervical cancer. Most importantly, selected E6/E7-specific siRNA candidates in combination with RT enhanced the anti-tumor effects in cervical carcinomas.

\section{Results and Discussion}

\subsection{Effect of HPV18 E6/E7-Specific Lead siRNAs in Combination with Radiation on Cervical Cancer Cells}

In a previous study, we revealed that E6/E7-specific siRNA, silencing both $E 6$ and $E 7 \mathrm{mRNA}$, was more efficacious than E6-specific siRNA [17]. Moreover, the combination of E6/E7-specific siRNA and CDDP had a greater therapeutic efficacy in cervical cancer cells. The aim of this study was to identify siRNAs that have the potential to silence both HPV18- and 16-type E6/E7 mRNA and simultaneously decrease E6/E7 protein-mediated degradation of TP53 in cervical cancer cells. A list of HPV18- and 16-type E6/E7 siRNA target sequences was generated (Tables S1 and S2). Ten library HPV-siRNAs were generated and screened for their silencing effects on HPV18, as well as HPV16-type E6/E7, respectively. The inhibition efficiency of siRNAs (103, 426, 450, 456 and 458) was shown by their significant anti-proliferative effect, unlike that of the other siRNAs (Figure S1a). To identify potential therapeutic siRNA, five out of 10 siRNAs were subjected to further analysis. As shown in Figure 1A, the inhibition of HeLa cell growth by at least $70 \%$ was achieved even at a low concentration. We also determined the cellular protein expression levels of E7, as well as TP53 in HeLa cells after E6/E7 silencing by siRNAs. With regard to TP53 and E7 protein levels, we found that siRNA 426 or 450 was able to silence E6/E7 expression more effectively than the other siRNAs (Figure 1B). Our results indicate that siRNA 426 and 450 showed a more robust effect than the other siRNAs did, in a dose-dependent manner (Figure S1b,c). After systematic screening of the library in triplicate, these results demonstrate that new, highly potent HPV18 siRNAs termed 426 and 450 are capable primary lead siRNAs. Similarly, on our screening analysis in SiHa cells (Figure S1c), HPV16-type-specific lead siRNAs termed 366 and 448 were selected along with siRNA 497 [16] for further studies. 
A

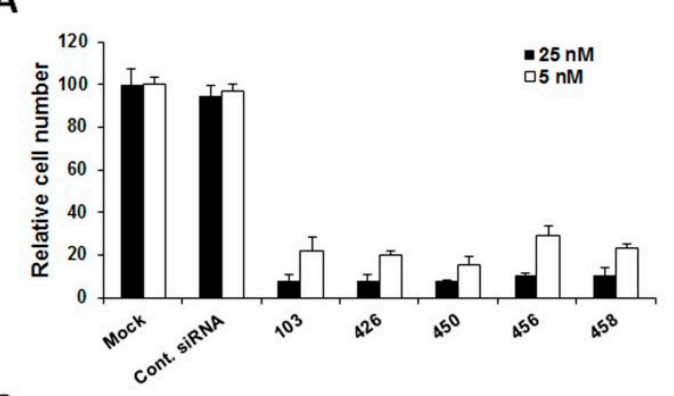

C

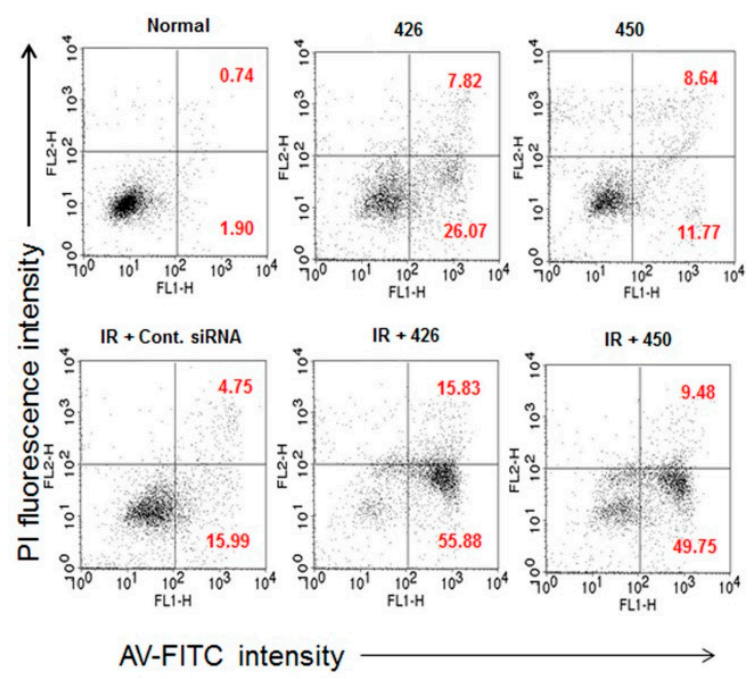

$\mathrm{E}$

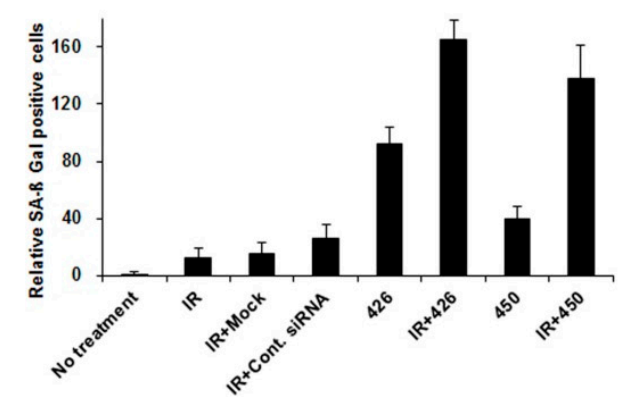

B

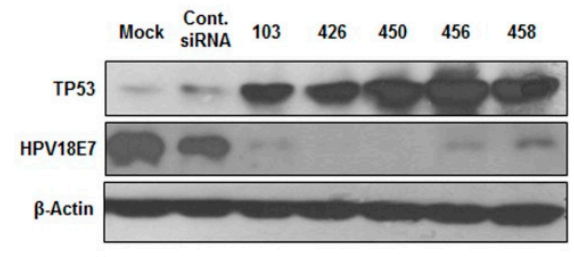

D
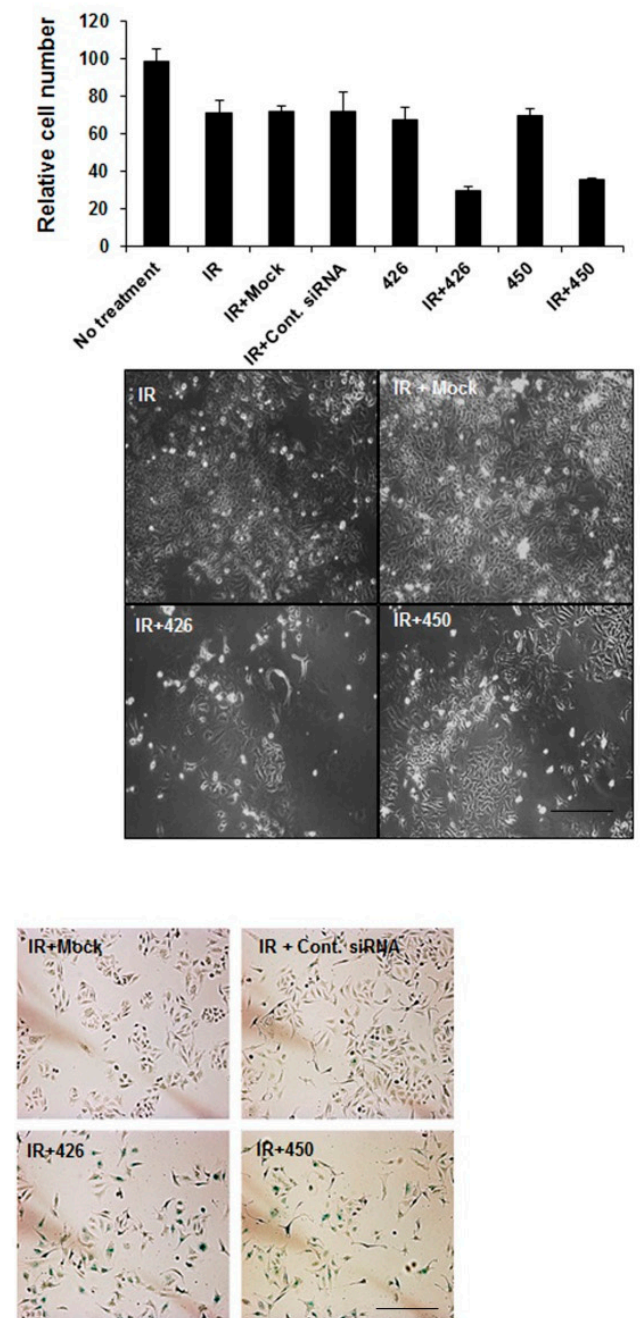

Figure 1. Screening and systematic analysis of HPV18 E6/E7-specific siRNA in combination with radiation. (A) Trypan blue assay showing the number of viable HeLa cells transfected with library siRNAs $(103,426,450,456$ and 458). In these studies, HeLa cells were transfected with 5 or $25 \mathrm{nM}$ of each siRNA. The number of cells was compared to reagent alone without siRNAs (mock); (B) Changes in TP53 and HPV18 E7 expression levels in HeLa cells following transfection with HPV18 E6/E7-specific library siRNAs were detected by Western blotting. $\beta$-actin was used as a loading control; (C) Annexin-V binding assay showing the percentage of apoptotic HeLa cells transfected with siRNA 426 or 450 for $28 \mathrm{~h}$ or siRNAs in combination with $\gamma$-irradiation; (D) The effects of E6/E7-specific siRNA 426 or 450 in combination with $\gamma$-irradiation on cell viability and morphology are shown. Scale bar: all are $200 \mu \mathrm{m}$; and (E) The effect of HPV E6/E7-specific siRNAs alone or combined with $\gamma$-irradiation (IR) on cellular senescence, Scale bar: all are $200 \mu \mathrm{m}$. 
Next, we have validated and compared the silencing effect of lead siRNAs in combination with radiation. We determined the silencing effect of HPV18 E6/E7-specific siRNA 426 or 450 in combination with radiation on apoptosis, cell viability and cellular senescence. HeLa cells were irradiated ( 2 Gy), transfected with siRNA 426 or 450, collected after a three-day exposure to the agents and analyzed by a flow cytometer. The percentages of Annexin-V-positive apoptotic cells are summarized in Figure 1C. siRNA 426 or 450, but not control siRNA, in combination with irradiation, significantly increased the percentage of Annexin-V-positive apoptotic HeLa cells. The apoptosis of cells transfected with siRNA 426 or 450 in combination with irradiation was significantly higher than that of the cells transfected with siRNAs alone. As shown in Figure 1D, growth of the cells receiving irradiation in the presence of siRNA 426 or 450 was significantly higher, and the cells showed morphological changes. Next, senescence-associated $\beta$-galactosidase (SA- $\beta$-Gal) activity was assessed three days after exposure to the combined-treatment of E6/E7-specific siRNAs with irradiation. Significantly higher SA- $\beta$-Gal activity was observed in the HeLa cells transfected with siRNA 426 or 450 in combination with irradiation, as observed by strong blue staining (Figure 1E). However, transfection of HeLa cells with E6/E7-specific siRNAs alone resulted in moderate SA- $\beta$-Gal activity. The ratio of SA- $\beta$-Gal-positive cells was summarized. Therefore, E6/E7-specific siRNA 426 or 450 in combination with radiation increased the cellular senescence of cervical cancer cells. Similar effects were observed with the HPV16-type-specific siRNA 497 (Figure S2). These results suggest that E6/E7-specific siRNAs augment the cytotoxicity of target tumor cells by radiation.

To determine the synergistic, additive or antagonistic effects of combination treatment of E6/E7-specific siRNAs with irradiation in HeLa cells, Chou-Talalay analysis was used. The synergistic activity of HPV18 E6/E7-specific siRNA 426 (5 or $25 \mathrm{nM}$ ) and HPV16 E6/E7-specific siRNA 497 (25 or $50 \mathrm{nM}$ ) with irradiation (2, 4 or 4.8 Gy) was demonstrated to be statistically significant (Table 1). Therefore, these findings confirm that E6/E7-specific siRNAs synergized with radiation to induce a therapeutic effect on cervical cancer cells.

Table 1. The synergistic therapeutic effect of siRNA 426 or siRNA 497 in combination with $\gamma$-irradiation was demonstrated by Chou-Talalay analysis.

\begin{tabular}{cccc}
\hline 426 siRNA (nM) & \multicolumn{1}{c}{ Radiation (Gy) } & Cytotoxicity (\%) & CI * \\
\hline \multicolumn{4}{c}{ HPV18-Type HeLa Cells } \\
\hline 5 & 4.8 & 86 & 0.73 \\
25 & 4.8 & 83 & 0.86 \\
5 & 2 & 43 & 0.9 \\
25 & 2 & 51 & 0.9 \\
\hline 497 siRNA (nM) & Radiation (Gy) & Cytotoxicity (\%) & CI * \\
\hline \multicolumn{5}{c}{ HPV16-Type SiHa Cells } \\
\hline 50 & 2 & 78 \\
50 & 2 & 68 & 0.63 \\
50 & 4 & 77 & 0.71 \\
& * Combination index (CI) <1 indicates synergism.
\end{tabular}




\subsection{In Vivo Effects of HPV18 E6/E7-Specific siRNA in Combination with Radiation}

To examine the efficacy of HPV18 E6/E7-specific siRNA in combination with radiation therapy in the treatment of established human cervical cancer, luciferase-transfected HeLa cells were injected subcutaneously into the hind legs of nude mice (Figure 2A). Five days later, human tumor xenograft mice were injected intravenously with siRNA 426 six times at two-day intervals. On the day after the first siRNA 426 injection, the mice were irradiated with 8 Gy. As shown in Figure $2 \mathrm{~B}$, the mice receiving injections of siRNA 426 alone, control GFP-siRNA or untreated controls all developed tumors within 11 days after tumor challenge. However, three out of five mice receiving irradiation alone survived without tumor burden to the end of the experimental period (Day 21). The combination therapy of siRNA 426 with irradiation generated a superior therapeutic effect against established tumors compared with irradiation alone $(p=0.016)$. In the combination-treated group, all of the mice survived without tumor burden. Based on in vivo imaging, the combination therapy group, but not control groups, maintained low luciferase activity throughout the treatment period. These results show that HPV18 E6/E7-specific siRNA in combination with radiation significantly decreased tumor growth in a mouse xenograft model of human cervical cancer. For more dramatic in vivo efficacy, further studies are required to develop the siRNA nanoparticles that have specific targeted delivery systems [26-29].

Tumors treated with radiation therapy alone and combined with siRNA 426 were well demarcated and generally homogenous, with low cellular polymorphism. By contrast, tumors treated with green fluorescent protein (GFP) siRNA showed infiltrative margins, increased heterogeneity and high cellular polymorphism (Figure 2C). Consistent with these observations, TP53- and TUNEL-positive cells were markedly higher in the combination-treated mice than in the animals treated with GFP siRNA.
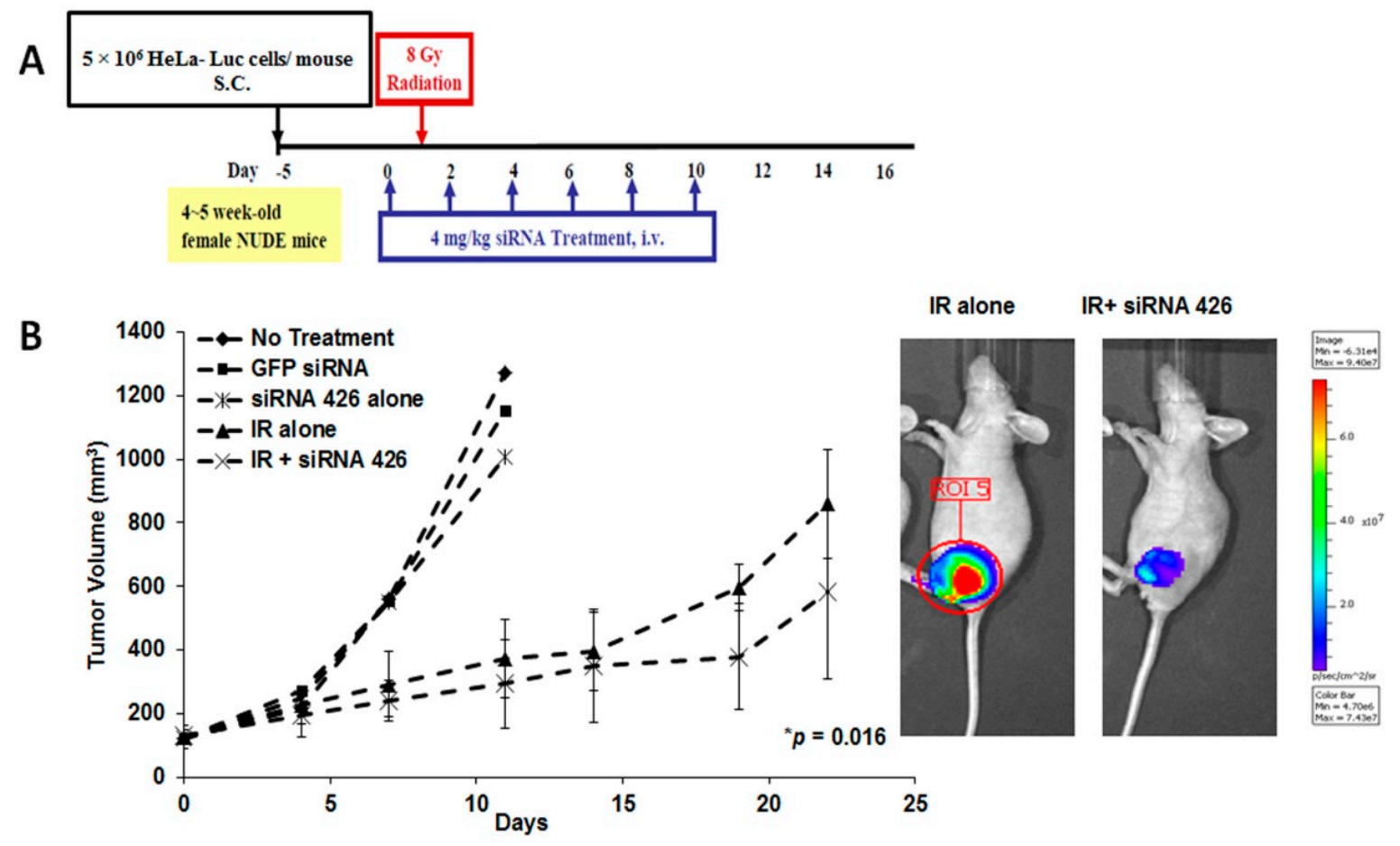

Figure 2. Cont. 

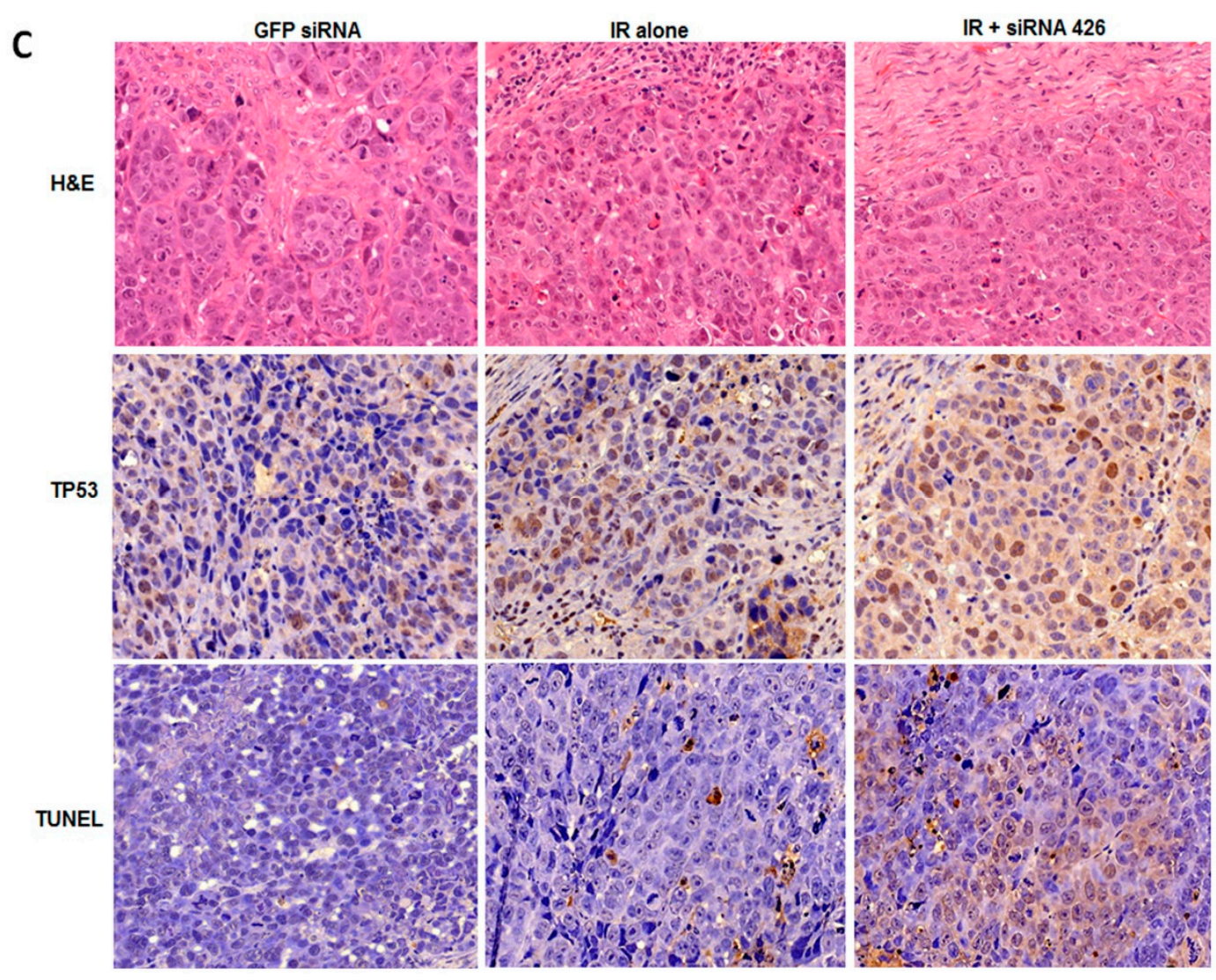

Figure 2. In vivo therapeutic effect of E6/E7-specific siRNAs in combination with radiation in human cervical cancer xenografted mice. (A) Treatment protocol; and (B) Luciferase activity was measured in live mice using the Luminescence Animal Imaging System, and tumor images were taken after 19 days. Untreated or control GFP-specific siRNA-treated mice served as controls. ${ }^{*} p=0.016$ at Day 19 , compared with $\gamma$-irradiation alone; and $(\mathbf{C})$ H\&E staining $(\times 400)$, TP53 $(\times 400)$ and TUNEL immunohistochemical (IHC) $(\times 400)$ analysis on Day 25.

\subsection{The Effect of Chemically-Modified E6/E7-Specific siRNAs on Silencing Efficiency and Serum Stability}

Identification of chemically-modified potent siRNA is crucial for the therapeutic application of siRNA technology. Earlier reports have shown that 2'-O-methyl (2'-OMe) and 2'-fluoro (2'-F) modifications to siRNAs and Dicer-substrate RNAs can have significant effects on potency and serum stability without the loss of RNAi activity [30-32]. To increase the activity and stability of HPV E6/E7-specific siRNA, we designed and chemically modified (2'-OMe, 2'-F) the siRNAs (Table 2). Moreover, we examined the silencing efficiency and serum stability of the modified siRNAs.

Based on our results, HPV18-type lead siRNAs (426 and 450) and HPV16-type lead siRNAs (366, 448 and 497) were chemically modified to enhance the nuclease stability. In our first line investigation, we examined the stability of siRNAs and the silencing efficiency by introducing 2'-OMe and $2^{\prime}-\mathrm{F}$ modifications. As shown in Figure 3A, the modified derivatives of siRNA 426 or 497 (Table 2) decreased the proliferation of $\mathrm{HeLa}$ or SiHa cells. In particular, cells receiving siRNA 426_d5 (derivative 5) and siRNA 497_d2 (derivative 2) showed significant cell growth inhibition 
compared to the other derivatives. We further assessed the efficiency of modified E6/E7-specific siRNAs by investigating the changes in TP53 and E7 protein levels. siRNA 426_d5 and siRNA 497_d2 were shown to affect the expression levels of TP53 and E7 (Figure 3B). Interestingly, their derivatives also significantly decreased E6 mRNA levels (Figure 3C) and increased CDKN1A mRNA levels (Figure 3D). We next evaluated the serum stability of chemically-modified siRNAs by using gel electrophoresis analysis. The chemically-modified derivatives were comparatively more stable than the unmodified siRNAs (Figure 3E). We simultaneously determined the efficacy of other HPV16 E6/E7-specific modified siRNAs $(366,448)$ and HPV16 E6/E7-specific siRNA 450 (Supplementary Table S3). Among the modified derivatives, siRNA 450_d4 (derivative 4), siRNA 366_d2 (derivative 2) and siRNA 448_d2 (derivative 2) were selected as candidates based on the experimental results (Figures S3-S5). Similarly, in flow cytometric analysis, modified siRNAs transfection resulted in an increased percentage of apoptotic cells (Figure S6).

Table 2. List of selected siRNA derivatives used for chemical modification.

\begin{tabular}{|c|c|c|c|c|}
\hline Name & & ivative Number & Sequence & Selected Candidates \\
\hline \multirow{14}{*}{ HPV18-type siRNA 426 (19mer) } & \multirow{2}{*}{1} & Sense (S) & 5'-caaccgagcacgacaggaa dTdT-3' & \\
\hline & & Antisense (AS) & 5'-uuccugucgugcucgguug dTdT-3' & \\
\hline & \multirow{2}{*}{2} & $6 \mathrm{FC}(\mathrm{S})$ & 5'-caaccgagcacgacaggaa dTdT-3' & \\
\hline & & Antisense (AS) & 5'-uuccugucgugcucgguug dTdT-3' & \\
\hline & \multirow{2}{*}{3} & $6 \mathrm{FC}(\mathrm{S})$ & 5'-caaccgagcacgacaggaa dTdT-3' & \\
\hline & & $4 \mathrm{MeU}(\mathrm{AS})$ & 5'-uuccuguncgugecucgguug dTdT-3' & \\
\hline & \multirow{2}{*}{4} & $6 \mathrm{FC}(\mathrm{S})$ & 5'-caaccgagcacgacaggaa dTdT-3' & \\
\hline & & $6 \mathrm{MeU}(\mathrm{AS})$ & $5^{\prime}$-uuccugucgugugcucgguug dTdT-3' & \\
\hline & \multirow{2}{*}{5} & $5 \mathrm{MeG}(\mathrm{S})$ & 5'-caaccgagcacgacaggaa dTdT-3' & \multirow{2}{*}{ d5 } \\
\hline & & Antisense (AS) & 5'-uuccugucgugcucgguug dTdT-3' & \\
\hline & \multirow{2}{*}{6} & $5 \mathrm{MeG}(\mathrm{S})$ & 5'-caaccgagcacgacaggaa dTdT-3' & \\
\hline & & $4 \mathrm{MeU}(\mathrm{AS})$ & 5'-uuccuguncguggcucgguug dTdT-3' & \\
\hline & 7 & $5 \mathrm{MeG}(\mathrm{S})$ & 5'-caaccgagcacgacaggaa dTdT-3' & \\
\hline & 1 & $6 \mathrm{MeU}(\mathrm{AS})$ & 5'-uuccugucgugcucgguug dTdT-3' & \\
\hline \multirow{8}{*}{ HPV16-type siRNA 497 (21mer) } & \multirow{2}{*}{1} & Sense $(\mathrm{S})$ & 5'-gaccggucgauguaugucuug-3' & \\
\hline & & Antisense (AS) & $5^{\prime}$-agacauacaucgaccggucca-3' & \\
\hline & \multirow{2}{*}{2} & Sense (S) & 5'-gaccggucgauguaugucuug-3' & \multirow{2}{*}{$\mathrm{d} 2$} \\
\hline & & $3 \mathrm{MeU}(\mathrm{AS})$ & 5'-agacauacaucgaccggụcca-3' & \\
\hline & \multirow{2}{*}{3} & $6 \mathrm{MeU}(\mathrm{S})$ & $5^{\prime}$-gaccggucgauguauuggucuuug-3' & \\
\hline & & Antisense (AS) & 5'-agacauacaucgaccggucca-3' & \\
\hline & \multirow{2}{*}{4} & $6 \mathrm{MeU}(\mathrm{S})$ & $5^{\prime}$-gaccggucgauguaugucuug-3' & \\
\hline & & $3 \mathrm{MeU}(\mathrm{AS})$ & $5^{\prime}$-agacauacaucgaccggucca-3' & \\
\hline
\end{tabular}

Lower case letters represent ribonucleotides; Underlines are methyl modifications (2'-OMe); Bold italics are F-fluoro modifications ( $\left.2^{\prime}-\mathrm{F}\right)$; S, sense strand; AS, antisense strand. 
A

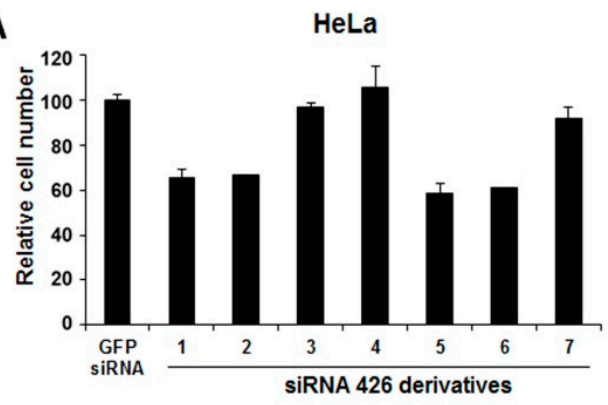

B

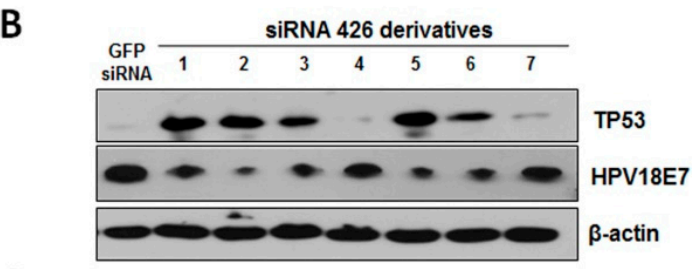

C $\overline{\mathrm{s}}$

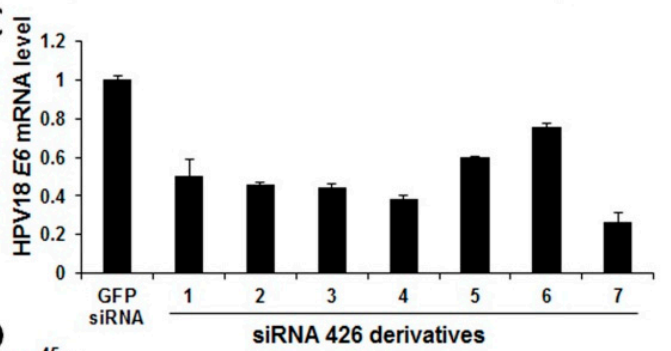

D

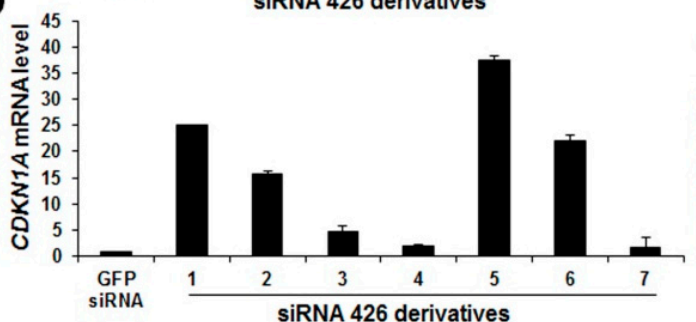

$\mathbf{E}$

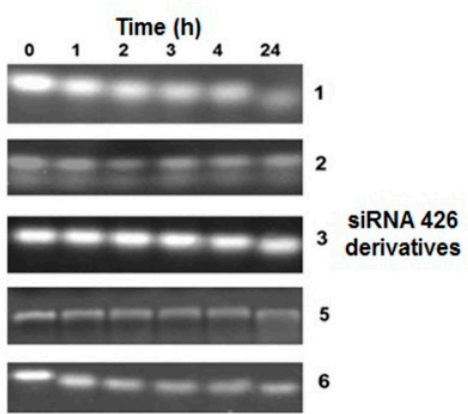

$\mathrm{SiHa}$
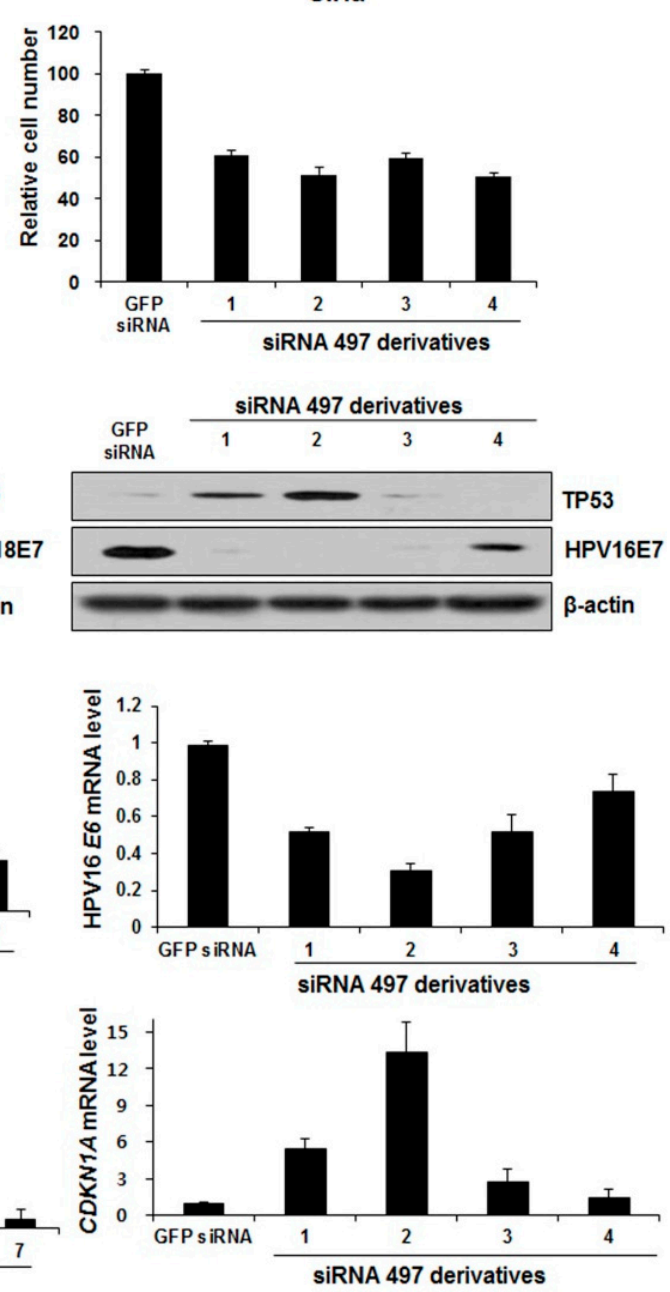

Time (h)

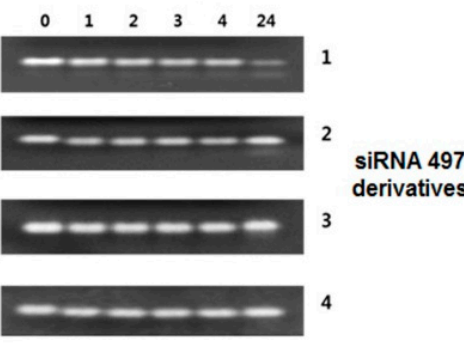

Figure 3. Determining the stability and silencing activities of chemically-modified derivatives of HPV16- and 18 E6/E7-specific lead siRNAs. (A) Trypan blue assay showing the number of viable HeLa cells transfected with 2'-OMe-modified derivatives of siRNA 426 and SiHa cells transfected with 2'-OMe modified derivatives of siRNA 497. GFP-specific siRNA (control siRNA) served as controls; (B) Silencing efficiency of 2'-OMe-modified siRNA derivatives on E7 expression and changes in TP53 expression were also analyzed by Western blotting. $\beta$-actin was used as a loading control; (C) E6 and (D) CDKN1A mRNA expression as determined by qRT-PCR; and (E) Gel electrophoresis analysis showing the serum stability of 2'-OMe-modified siRNA derivatives. Unmodified (Lane 0 ) and modified siRNA 426 or 497 derivatives were analyzed by electrophoresis on $15 \%$ native polyacrylamide gels. 


\subsection{Synergistic Effect of E6/E7-Specific siRNA Pool in Combination with Radiation on Cervical Cancer Cells}

In nature, the siRNAs generated by Dicer represent a pool of siRNAs that function together to silence gene expression. Pooled siRNAs generated in this manner are efficient silencers and have low off-target effects. We evaluated the apoptotic efficacy of HPV18-type siRNA pool (SP) using $10 \mathrm{nM}$ of each siRNA and HPV16-type siRNA pool (SP) at 7 and $10 \mathrm{nM}$, because the preferred method to verify the identified siRNA candidates in screens by utilizing SP is to increase the efficiency of primary candidates. Interestingly, in both $\mathrm{HeLa}$ and SiHa cells, SP candidates could efficiently induce apoptosis even at a low concentration (Figure S7a).

To assess whether the E6/E7-specific pooled siRNAs promote cell death and senescence, we first tested and compared the cytotoxicity of cervical cancer cells. Irradiated (2 Gy) HeLa or SiHa cells were transfected with pooled HPV E6/E7-specific siRNAs and analyzed after three-days' exposure. SP-transfected HeLa or SiHa cells without irradiation served as the controls. As shown in Figure 4A, HPV18 E6/E7-specific SP (426_d5 and 450_d4) led to significantly higher growth inhibition than either siRNA 426_d5 or 450_d4 alone did. Pooled siRNAs in combination with irradiation significantly enhanced growth inhibition to levels greater than observed with other treatments. Similar results were observed in SiHa cells transfected with HPV16 E6/E7-specific SP (366_d2, 448_d2 and 497_d2).

We investigated the expression levels of TP53, HPV E7 (Figure 4B) and p16INK4a (Figure S7b). Irradiation with SP significantly induces the expression of TP53 and lowers the expression of p16INK4A and E7 than SP without irradiation and irradiation alone did. The CDKN1A mRNA level was increased substantially in SP-transfected cells with irradiation (Figure S7c). Furthermore, we observed and compared the effect of SP on apoptosis and cellular senescence in cervical cancer cells. SP alone and/or in combination with irradiation significantly increased the percentages of Annexin-V-positive apoptotic HeLa cells. The percentages of Annexin-V-positive apoptotic cell are summarized in Figure 4C. The degree of apoptosis in cells treated with pooled siRNAs in combination with irradiation was significantly higher than in cells treated with either siRNA 426_d5 or 450_d4 alone. In addition, siRNA 366_d2, 448_d2 and 497_d2 in combination with irradiation also increased the percentages of Annexin-V-positive cells in HPV16-positive SiHa cells. SA- $\beta$-Gal activity was assessed to determine the cellular senescence (Figure S7d), and the relative ratio of positive senescence cells is summarized in Figure 4d. The pooled siRNAs in combination with irradiation promoted higher cellular senescence in HeLa and SiHa cells. Moreover, the SA- $\beta$-Gal activity in pooled siRNA-transfected cells was significantly higher than in cells transfected with candidate siRNA alone. All of these results suggest that E6/E7-specific SP efficiently induced the cytotoxicity, apoptosis and cellular senescence of cervical cancer cells. Therefore, the low concentration of each siRNA in pooled siRNAs with radiation strongly inhibited the growth of cervical cancer cells.

Despite progress in the treatment of cervical cancer by using conventional chemotherapies and therapeutics in combination with concurrent radiation, this disease still remains fatal in the majority of patients. Thus, new therapeutic strategies are required to improve therapeutic options. The specific siRNAs targeting HPV-encoded oncogenes have definite therapeutic benefits in patients with cervical cancer. Earlier, we identified and validated some HPV18- and 16-type E6/E7-specific siRNA hits as 
chemosensitizers. Thus, we have established a proof-of-concept of combination therapy with E6/E7-specific siRNAs and CDDP [17].
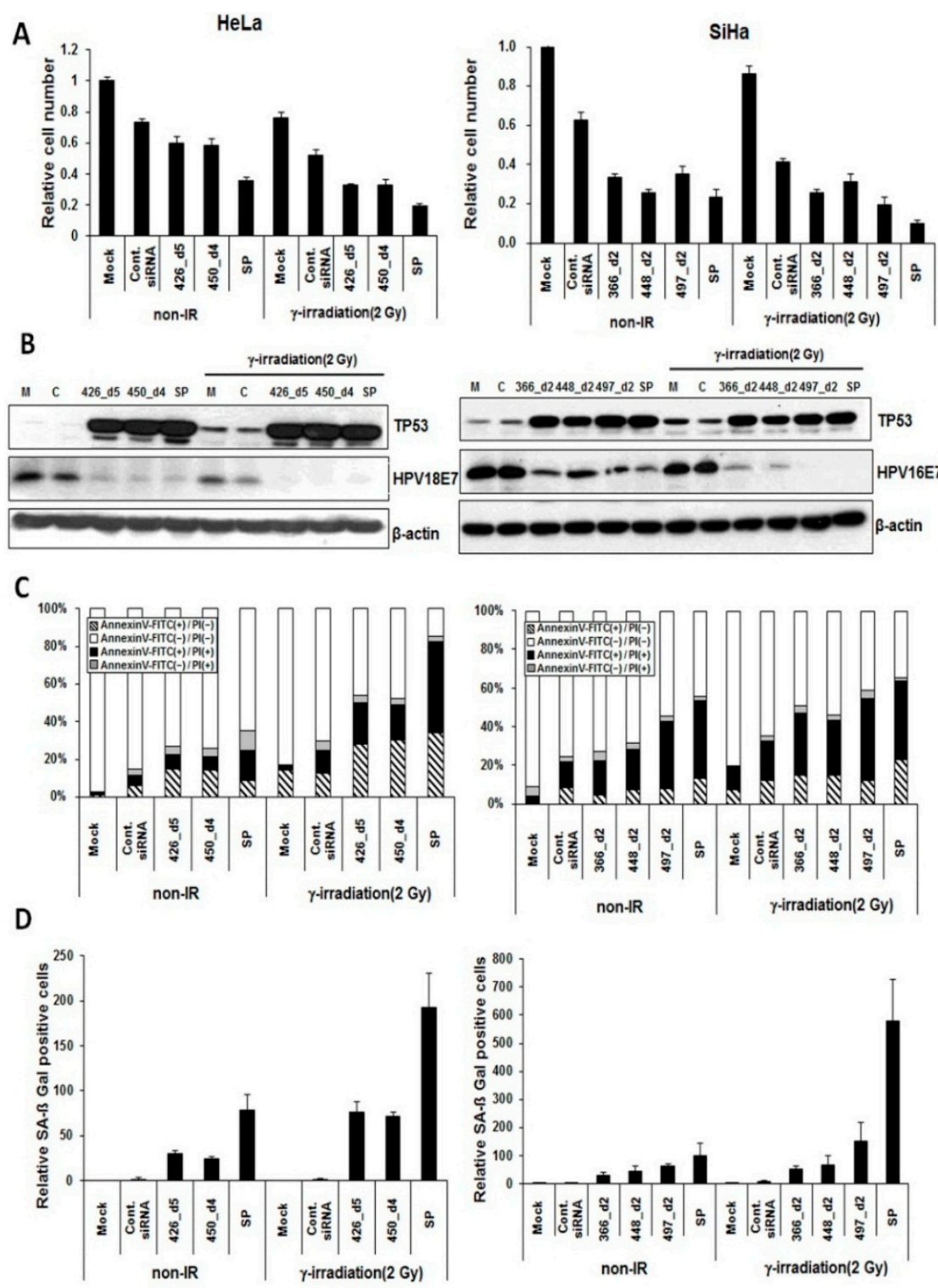

Figure 4. Synergistic effect of E6/E7-specific siRNA pools in combination with radiation on cervical cancer cells. HeLa cells were transfected with $20 \mathrm{nM}$ of each selected siRNA derivative or siRNA pools (SP; $10 \mathrm{nM}$ siRNA each) or in combination with $\gamma$-irradiation. Similarly, SiHa cells were transfected with $20 \mathrm{nM}$ of each selected siRNA derivative or SP (7 nM siRNA each) or in combination with $\gamma$-irradiation. The number of cancer cells was determined by siRNAs alone (non-IR) or siRNAs in combination with $\gamma$-irradiation. Untransfected and control siRNA served as controls. (A) Trypan blue assay showing the number of viable cells transfected with siRNA pools, siRNA alone and in combination with $\gamma$-irradiation; (B) Western blotting analyses showing the expression of TP53 and HPV E7 proteins. M, mock; C, control siRNA; SP, siRNA pool; (C) Annexin-V and PI binding assay showing the percentages of apoptotic cells transfected with selected siRNA derivatives; and (D) The effect of HPV E6/E7-specific siRNAs on cellular senescence is also shown. 
According to the RNAi therapeutics pipeline, further studies were performed to discover highly potent E6/E7-specific lead siRNAs through siRNA library screenings. The anti-cancer effects may be more profound from those found in previous studies if more potent E6/E7-specific siRNAs are selected. In fact, newly identified siRNAs in this study elicited much stronger effects than E6/E7-specific siRNA hits, despite short-term exposure at low concentrations of siRNAs. Finally, five lead HPV18- and 16-type-specific siRNAs were selected, for which only siRNA 497 sequences were modified as an RNA-DNA chimera to enhance the target specificity in a previous study [33]. In addition, highly potent siRNAs, which were rationally designed, screened, validated for efficacy and chemically modified for stability, can mimic the pools generated in nature. These siRNA pools reduce the effective concentration and off-target effects of each siRNA, because each siRNA has a strong therapeutic effect. In this study, we have also used the pooled siRNAs to promote and accelerate the growth inhibition of cervical cancer cells. Our results confirmed that the pooled siRNAs in combination with radiation strongly inhibited the growth of cervical cancer cells. Therefore, the therapeutic benefit of highly potent siRNA pools means that they are of significant interest for drug development.

In the case of cervical cancer, CCRT is recommended as the first-line therapy. While this practice guideline has improved the survival rates, CCRT treatment fails in the majority of patients with residual cancers, adverse effects, local recurrences or distance recurrences common [21-23]. Therefore, there remain medically unmet needs for cervical cancer. First, in the case of early lesion recurrence and early lesions with high risk factors after surgery, such as margin or lymph node metastasis and microscopic parametrical involvement, as well as in advanced lesions beyond stage II $\mathrm{B}$, it is important to maximize the effectiveness of CCRT and pelvic radiation in order to decrease recurrence and improve the survival rate. Next, there are also unmet needs for patients who have disease recurrence and metastasis after failure of CCRT. Clinical studies have been conducted to improve progression-free survival, overall survival and the reduction of severe adverse events [34-37]. Thus, to meet these medical needs, further studies are needed to evaluate combination therapy with CCRT and E6/E7-specific siRNAs.

\section{Experimental Section}

\subsection{Cell Lines and Western Blotting}

HeLa cells are an HPV18-positive human cervical cancer cell line. HeLa-Luc, a stably-transfected and bioluminescent human tumor cell line derived from HeLa cells, was obtained from Xenogen Corp. (Alameda, CA, USA). The HPV16-positive human cervical cancer cell line SiHa was obtained from the American Type Culture Collection (Manassas, VA, USA) with its identity verified by short tandem repeat analysis. The cells were routinely tested for mycoplasma. Primary antibodies against TP53, E7, p16INK4A and $\beta$-actin were used for Western blotting (for details, see Supplementary Materials and Methods). 


\subsection{In Vitro Models of siRNA Transfection and $\gamma$-Irradiation}

siRNAs against HPV18 E6/E7 (426, 450), HPV16 E6/E7 (366, 448, 497) and control siRNA were synthesized from BIONEER (Dae-joen, Korea). Transfection was performed with siRNA using DharmaFect (Dharmacon, Lafayette, CO, USA), according to the manufacturer's instruction. Chemically-modified (2'-sugar modification by 2'-OMe, 2-F') siRNA duplex derivatives were designed and synthesized by BIONEER (Dae-joen, Korea). All of the modified siRNA duplexes are complementary to the target sequences that are shown in Table 2. All siRNAs were suspended in DEPC-treated water to a final concentration of $5 \mu \mathrm{g} / \mu \mathrm{L}$. For irradiation, the cells were seeded at a density of $5 \times 10^{5}$ cells $/ 100 \mathrm{~mm}$ dish. After $24 \mathrm{~h}$, the cells were irradiated with 2-3 Gy of $\gamma$-irradiation in a GC 3000 Elan irradiator (MDS Nordion, Ottawa, ON, Canada). The following day, the cells were trypsinized and re-plated at a density of $3 \times 10^{5}$ cell $/ 100-\mathrm{mm}$ dish.

\subsection{Flow Cytometric Analysis and Senescence-Associated B-Galactosidase Assay}

Apoptotic cells were stained with Annexin V-fluorescence isothiocyanate (FITC) and propidium iodide (BD PharMingen, San Diego, CA, USA), according to the manufacturer's instructions, and were quantified by flow cytometry. For senescence analysis, the cells were fixed in $2 \%$ formaldehyde/ $0.2 \%$ glutaraldehyde and stained at $\mathrm{pH} 6.0$ by using X-Gal (5-bromo-4-chloro-3-indolyl- $\beta$-Dgalactopyranoside), by the method described previously [13]. SA- $\beta$-Gal-positive cells were counted in three representative fields.

\subsection{Quantitative Real-Time PCR Analysis}

Following single-agent or combination therapy, the expression of HPV18 E6, HPV16 E6 and CDKN1A (p21 $1^{\text {cip1})}$ was quantified using TaqMan quantitative real-time PCR (qRT-PCR) analysis (for details, see Supplementary Materials and Methods).

\subsection{Serum Stability}

siRNA duplexes $(9 \mu \mathrm{g})$ were incubated at $37{ }^{\circ} \mathrm{C}$ in $90 \mu \mathrm{L} 10 \%$ human serum. Aliquots of $12 \mu \mathrm{L}$ were taken at various time points $(0,1,2,3,4$ and $24 \mathrm{~h})$ and immediately frozen at $-70{ }^{\circ} \mathrm{C}$. RNAs were separated using $15 \%$ polyacrylamide-TBE under non-denaturing conditions and were photographed on a UV-transilluminator, after performing the EtBr staining [38].

\subsection{In Vivo Xenografts, Imaging and Immunohistochemical Analysis}

Balb/c-nude mice were challenged with $5 \times 10^{6}$ luciferase-transfected HeLa cells into the hind legs. After five days, cationic liposome/siRNA ( $4 \mathrm{mg} / \mathrm{kg}$ body weight, $100 \mu \mathrm{L}$ ) was injected into the tail vein once every $48 \mathrm{~h}$. The details of the cationic liposome preparation are in the Supplementary Materials and Methods. On the day after the first siRNA 426 injection, irradiation was given to the mice at 8 Gy. Tumors were visualized, and luminescence was measured 14-15 days post-implantation, using region-of-interest analysis and the in vivo imaging system (IVIS; Xenogen Corp., Alameda, CA, USA). This study was approved by the Institutional Review Board of Asan Medical Center (No. 2009-13-131). 
Formalin-fixed and paraffin-embedded tumors were sectioned and stained with hematoxylin and eosin (H\&E) or immunostained by using anti-TP53 antibodies. The terminal deoxynucleotidyl transferase (TdT)-mediated dUTP nick-end labeling (TUNEL) assay was performed to detect apoptosis (for details, see Supplementary Materials and Methods)

\subsection{Chou and Talalay Analysis}

To determine the pharmacological interaction between siRNA and irradiation, the Chou and Talalay analysis was used [24]. This method assesses synergy and antagonism by quantifying the divergence of the combination effect from the expected additive effect of the two therapeutic agents. A combination index (CI) was estimated from the dose-effect data. A CI of $<1,=1$ and of $>1$ indicates synergy, additive effects and antagonism, respectively.

\section{Conclusions}

We have confirmed the therapeutic synergy in vitro and in vivo between chemically-modified HPV18 or HPV16 E6/E7-specific siRNAs and radiation in cervical cancer. In addition, we confirmed the radiosensitizer effect of our HPV18 E6/E7-specific siRNA in vivo. Therefore, E6/E7-specific siRNA candidates have therapeutic potential as radiosensitizers in cervical cancer. Pooled HPV E6/E7-specific siRNAs in combination with radiation are more potent therapeutics than single siRNA to promote an anti-tumor effect in HPV-positive cervical cancer. Further work is warranted, encompassing the optimization of a suitable delivery system to improve cellular siRNA uptake for better therapeutic effect. To our knowledge, this is the first study showing the synergistic therapeutic effect of HPV E6/E7-specific siRNAs in combination with radiation for the treatment of cervical cancer. This information will be important for improving strategies involving siRNA therapeutics for treating patients with cervical cancer and possibly other tumors relating to HPV.

\section{Supplementary Materials}

Supplementary materials can be found at http://mdpi.com/1422-0067/16/06/12243/s1.

\section{Acknowledgments}

This work was supported by the National Research Foundation of Korea grant for the Global Core Research Center (GCRC) funded by the Korean government (MSIP) No. 2011-0030001 (Young Kee Shin), Samsung Biomedical Research Institute Grant SBRI C-B1-115-1 (Sang Yong Song), the Small \& Medium Business Administration No. S1069951 (Young Deug Kim) and the National Research Foundation of Korea Grant NRF-2013R1A1A2063951 (Sungyoul Hong).

\section{Author Contributions}

Hun Soon Jung, Nirmal Rajasekaran and Young Kee Shin conceived of and designed the study, collected data, performed data analysis and drafted the manuscript. Sang Yong Song, Young Deug Kim, Sungyoul Hong, Yoon-La Choi and Young Kee Shin collected data and contributed with valuable 
discussions. Hyuck Jae Choi and Young Seok Kim performed the radiation therapy and provided expert data interpretation. All authors read and approved the final manuscript.

\section{Conflicts of Interest}

Young Kee Shin owns stock in Abion Inc. Yoon-La Choi owns stock in Abion Inc. Young Deug Kim owns stock in Abion Inc. Hun Soon Jung owns stock options in Abion Inc. No potential conflicts of interest were disclosed by the other authors.

\section{References}

1. Huibregtse, J.M.; Scheffner, M.; Howley, P.M. Cloning and expression of the cDNA for E6-AP, a protein that mediates the interaction of the human papillomavirus E6 oncoprotein with p53. Mol. Cell. Biol. 1993, 13, 775-784.

2. Zur Hausen, H.; de Villiers, E.M. Human papillomaviruses. Annu. Rev. Microbiol. 1994, 48, 427-447.

3. Woodman, C.B.; Collins, S.I.; Young, L.S. The natural history of cervical HPV infection: Unresolved issues. Nat. Rev. Cancer 2007, 7, 11-22.

4. Hainaut, P.; Hernandez, T.; Robinson, A.; Rodriguez-Tome, P.; Flores, T.; Hollstein, M.; Harris, C.C.; Montesano, R. Iarc database of p53 gene mutations in human tumors and cell lines: Updated compilation, revised formats and new visualisation tools. Nucleic Acids Res. 1998, 26, 205-213.

5. Scheffner, M.; Munger, K.; Byrne, J.C.; Howley, P.M. The state of the $p 53$ and retinoblastoma genes in human cervical carcinoma cell lines. Proc. Natl. Acad. Sci. USA 1991, 88, 5523-5527.

6. Dyson, N.; Howley, P.M.; Munger, K.; Harlow, E. The human papilloma virus-16 E7 oncoprotein is able to bind to the retinoblastoma gene product. Science 1989, 243, 934-937.

7. DiMaio, D.; Petti, L.M. The E5 proteins. Virology 2013, 445, 99-114.

8. Maufort, J.P.; Shai, A.; Pitot, H.C.; Lambert, P.F. A role for HPV16 E5 in cervical carcinogenesis. Cancer Res. 2010, 70, 2924-2931.

9. Haasnoot, J.; Westerhout, E.M.; Berkhout, B. RNA interference against viruses: Strike and counterstrike. Nat. Biotechnol. 2007, 25, 1435-1443.

10. Jiang, M.; Milner, J. Selective silencing of viral gene expression in HPV-positive human cervical carcinoma cells treated with siRNA, a primer of RNA interference. Oncogene 2002, 21, 6041-6048.

11. Butz, K.; Ristriani, T.; Hengstermann, A.; Denk, C.; Scheffner, M.; Hoppe-Seyler, F. siRNA targeting of the viral E6 oncogene efficiently kills human papillomavirus-positive cancer cells. Oncogene 2003, 22, 5938-5945.

12. Hall, A.H.; Alexander, K.A. RNA interference of human papillomavirus type 18 E6 and E7 induces senescence in HeLa cells. J. Virol. 2003, 77, 6066-6069.

13. Yoshinouchi, M.; Yamada, T.; Kizaki, M.; Fen, J.; Koseki, T.; Ikeda, Y.; Nishihara, T.; Yamato, K. In vitro and in vivo growth suppression of human papillomavirus 16-positive cervical cancer cells by E6 siRNA. Mol. Ther. 2003, 8, 762-768.

14. Gu, W.; Putral, L.; Hengst, K.; Minto, K.; Saunders, N.A.; Leggatt, G.; McMillan, N.A. Inhibition of cervical cancer cell growth in vitro and in vivo with lentiviral-vector delivered short hairpin RNA targeting human papillomavirus E6 and E7 oncogenes. Cancer Gene Ther. 2006, 13, 1023-1032. 
15. Sima, N.; Wang, W.; Kong, D.; Deng, D.; Xu, Q.; Zhou, J.; Xu, G.; Meng, L.; Lu, Y.; Wang, S.; et al. RNA interference against HPV16 E7 oncogene leads to viral E6 and E7 suppression in cervical cancer cells and apoptosis via upregulation of $\mathrm{Rb}$ and $\mathrm{p} 53$. Apoptosis 2008, 13, 273-281.

16. Yamato, K.; Yamada, T.; Kizaki, M.; Ui-Tei, K.; Natori, Y.; Fujino, M.; Nishihara, T.; Ikeda, Y.; Nasu, Y.; Saigo, K.; et al. New highly potent and specific E6 and E7 siRNAs for treatment of HPV16 positive cervical cancer. Cancer Gene Ther. 2008, 15, 140-153.

17. Jung, H.S.; Erkin, O.C.; Kwon, M.J.; Kim, S.H.; Jung, J.I.; Oh, Y.K.; Her, S.W.; Ju, W.; Choi, Y.L.; Song, S.Y.; et al. The synergistic therapeutic effect of cisplatin with human papillomavirus E6/E7 short interfering RNA on cervical cancer cell lines in vitro and in vivo. Int. J. Cancer 2012, 130, 1925-1936.

18. Huang, H.; Huang, S.Y.; Chen, T.T.; Chen, J.C.; Chiou, C.L.; Huang, T.M. Cisplatin restores p53 function and enhances the radiosensitivity in HPV16 E6 containing SiHa cells. J. Cell. Biochem. 2004, 91, 756-765.

19. Chun, Y.J.; Park, I.C.; Park, M.J.; Woo, S.H.; Hong, S.I.; Chung, H.Y.; Kim, T.H.; Lee, Y.S.; Rhee, C.H.; Lee, S.J. Enhancement of radiation response in human cervical cancer cells in vitro and in vivo by arsenic trioxide $\left(\mathrm{As}_{2} \mathrm{O}_{3}\right)$. FEBS Lett. 2002, 519, 195-200.

20. Tsukamoto, H.; Shibata, K.; Kajiyama, H.; Terauchi, M.; Nawa, A.; Kikkawa, F. Aminopeptidase $\mathrm{N}(\mathrm{APV}) / \mathrm{CD} 13$ inhibitor, Ubenimex, enhances radiation sensitivity in human cervical cancer. BMC Cancer 2008, 8, 74 .

21. Keys, H.M.; Bundy, B.N.; Stehman, F.B.; Muderspach, L.I.; Chafe, W.E.; Suggs, C.L., III; Walker, J.L.; Gersell, D. Cisplatin, radiation, and adjuvant hysterectomy compared with radiation and adjuvant hysterectomy for bulky stage IB cervical carcinoma. N. Engl. J. Med. 1999, 340, 1154-1161.

22. Morris, M.; Eifel, P.J.; Lu, J.; Grigsby, P.W.; Levenback, C.; Stevens, R.E.; Rotman, M.; Gershenson, D.M.; Mutch, D.G. Pelvic radiation with concurrent chemotherapy compared with pelvic and para-aortic radiation for high-risk cervical cancer. N. Engl. J. Med. 1999, 340, $1137-1143$.

23. Rose, P.G.; Bundy, B.N.; Watkins, E.B.; Thigpen, J.T.; Deppe, G.; Maiman, M.A.; Clarke-Pearson, D.L.; Insalaco, S. Concurrent cisplatin-based radiotherapy and chemotherapy for locally advanced cervical cancer. N. Engl. J. Med. 1999, 340, 1144-1153.

24. Chou, T.C.; Talalay, P. Quantitative analysis of dose-effect relationships: The combined effects of multiple drugs or enzyme inhibitors. Adv. Enzym. Regul. 1984, 22, 27-55.

25. Peters, W.A., III; Liu, P.Y.; Barrett, R.J., 2nd.; Stock, R.J.; Monk, B.J.; Berek, J.S.; Souhami, L.; Grigsby, P.; Gordon, W., Jr.; Alberts, D.S. Concurrent chemotherapy and pelvic radiation therapy compared with pelvic radiation therapy alone as adjuvant therapy after radical surgery in high-risk early-stage cancer of the cervix. J. Clin. Oncol. 2000, 18, 1606-1613.

26. Shu, D.; Shu, Y.; Haque, F.; Abdelmawla, S.; Guo, P. Thermodynamically stable RNA three-way junction for constructing multifunctional nanoparticles for delivery of therapeutics. Nat. Nanotechnol. 2011, 6, 658-667.

27. Shu, Y.; Pi, F.; Sharma, A.; Rajabi, M.; Haque, F.; Shu, D.; Leggas, M.; Evers, B.M.; Guo, P. Stable RNA nanoparticles as potential new generation drugs for cancer therapy. Adv. Drug Deliv. Rev. 2014, 66, 74-89. 
28. Baumer, S.; Baumer, N.; Appel, N.; Terheyden, L.; Fremerey, J.; Schelhaas, S.; Wardelmann, E.; Buchholz, F.; Berdel, W.E.; Muller-Tidow, C. Antibody-mediated delivery of anti-KRAS-siRNA in vivo overcomes therapy resistance in colon cancer. Clin. Cancer Res. 2015, 21, 1383-1394.

29. Rychahou, P.; Haque, F.; Shu, Y.; Zaytseva, Y.; Weiss, H.L.; Lee, E.Y.; Mustain, W.; Valentino, J.; Guo, P.; Evers, B.M. Delivery of RNA nanoparticles into colorectal cancer metastases following systemic administration. ACS Nano 2015, 9, 1108-1116.

30. Czauderna, F.; Fechtner, M.; Dames, S.; Aygun, H.; Klippel, A.; Pronk, G.J.; Giese, K.; Kaufmann, J. Structural variations and stabilising modifications of synthetic siRNAs in mammalian cells. Nucleic Acids Res. 2003, 31, 2705-2716.

31. Allerson, C.R.; Sioufi, N.; Jarres, R.; Prakash, T.P.; Naik, N.; Berdeja, A.; Wanders, L.; Griffey, R.H.; Swayze, E.E.; Bhat, B. Fully 2'-modified oligonucleotide duplexes with improved in vitro potency and stability compared to unmodified small interfering RNA. J. Med. Chem. 2005, 48, 901-904.

32. Behlke, M.A. Chemical modification of siRNAs for in vivo use. Oligonucleotides 2008, 18, 305-319.

33. Yamato, K.; Egawa, N.; Endo, S.; Ui-Tei, K.; Yamada, T.; Saigo, K.; Hyodo, I.; Kiyono, T.; Nakagawa, I. Enhanced specificity of HPV16 E6E7 siRNA by RNA-DNA chimera modification. Cancer Gene Ther. 2011, 18, 587-597.

34. Garcia, A.A.; Blessing, J.A.; Vaccarello, L.; Roman, L.D.; Gynecologic Oncology Group, S. Phase II clinical trial of docetaxel in refractory squamous cell carcinoma of the cervix: A gynecologic oncology group study. Am. J. Clin. Oncol. 2007, 30, 428-431.

35. Long, H.J., III; Bundy, B.N.; Grendys, E.C., Jr.; Benda, J.A.; McMeekin, D.S.; Sorosky, J.; Miller, D.S.; Eaton, L.A.; Fiorica, J.V. Randomized phase III trial of cisplatin with or without topotecan in carcinoma of the uterine cervix: A gynecologic oncology group study. J. Clin. Oncol. 2005, 23, 4626-4633.

36. Sugiyama, T.; Yakushiji, M.; Noda, K.; Ikeda, M.; Kudoh, R.; Yajima, A.; Tomoda, Y.; Terashima, Y.; Takeuchi, S.; Hiura, M.; et al. Phase II study of irinotecan and cisplatin as first-line chemotherapy in advanced or recurrent cervical cancer. Oncology 2000, 58, 31-37.

37. Pectasides, D.; Fountzilas, G.; Papaxoinis, G.; Pectasides, E.; Xiros, N.; Sykiotis, C.; Koumarianou, A.; Psyrri, A.; Panayiotides, J.; Economopoulos, T. Carboplatin and paclitaxel in metastatic or recurrent cervical cancer. Int. J. Gynecolo. Cancer 2009, 19, 777-781.

38. Choung, S.; Kim, Y.J.; Kim, S.; Park, H.O.; Choi, Y.C. Chemical modification of siRNA to improve serum stability without loss of efficacy. Biochem. Biophys. Res. Commun. 2006, 342, 919-927.

39. Andersson, S.; Hansson, B.; Norman, I.; Gaberi, V.; Mints, M.; Hjerpe, A.; Karlsen, F.; Johansson, B. Expression of E6/E7 mRNA from "high risk" human papillomavirus in relation to cin grade, viral load and p16ink4a. Int. J. Oncol. 2006, 29, 705-711.

40. Ishikawa, M.; Fujii, T.; Saito, M.; Nindl, I.; Ono, A.; Kubushiro, K.; Tsukazaki, K.; Mukai, M.; Nozawa, S. Overexpression of p16 ink4a as an indicator for human papillomavirus oncogenic activity in cervical squamous neoplasia. Int. J. Gynecol. Cancer 2006, 16, 347-353.

41. Nielsen, G.P.; Stemmer-Rachamimov, A.O.; Shaw, J.; Roy, J.E.; Koh, J.; Louis, D.N. Immunohistochemical survey of p16ink4a expression in normal human adult and infant tissues. Lab. Investig. 1999, 79, 1137-1143. 
42. Klaes, R.; Friedrich, T.; Spitkovsky, D.; Ridder, R.; Rudy, W.; Petry, U.; Dallenbach-Hellweg, G.; Schmidt, D.; von Knebel Doeberitz, M. Overexpression of p16(ink4a) as a specific marker for dysplastic and neoplastic epithelial cells of the cervix uteri. Int. J. Cancer 2001, 92, 276-284.

43. Lau, W.M.; Ho, T.H.; Hui, K.M. P16ink4a-silencing augments DNA damage-induced apoptosis in cervical cancer cells. Oncogene 2007, 26, 6050-6060.

44. Van de Putte, G.; Holm, R.; Lie, A.K.; Trope, C.G.; Kristensen, G.B. Expression of p27, p21, and p16 protein in early squamous cervical cancer and its relation to prognosis. Gynecol. Oncol. 2003, $89,140-147$.

45. Munger, K.; Basile, J.R.; Duensing, S.; Eichten, A.; Gonzalez, S.L.; Grace, M.; Zacny, V.L. Biological activities and molecular targets of the human papillomavirus E7 oncoprotein. Oncogene 2001, 20, 7888-7898.

46. Mantovani, F.; Banks, L. The human papillomavirus E6 protein and its contribution to malignant progression. Oncogene 2001, 20, 7874-7887.

(C) 2015 by the authors; licensee MDPI, Basel, Switzerland. This article is an open access article distributed under the terms and conditions of the Creative Commons Attribution license (http://creativecommons.org/licenses/by/4.0/). 